\title{
Altered Spinal Motion in Low Back Pain Associated with Lumbar Strain and Spondylosis
}

\author{
Joseph S. Cheng ${ }^{1}$ Christopher B. Carr ${ }^{2}$ \\ Richard D. Komistek ${ }^{2}$ \\ 1 Department of Neurological Surgery, Vanderbilt University Medical \\ Center, Nashville, Tennessee, United States \\ ${ }^{2}$ Department of Biomedical Engineering, Center for Musculoskeletal \\ Research, University of Tennessee, Knoxville, Tennessee, United \\ States
}

Address for correspondence Joseph S. Cheng, MD, MS, Department of Neurological Surgery, Vanderbilt University Medical Center, T-4224 Medical Center North, Nashville, TN 37232, United States (e-mail: joseph.cheng@vanderbilt.edu).

Evid Based Spine Care J 2013;4:6-12.

\begin{abstract}
Study Design We present a patient-specific computer model created to translate twodimensional (2D) fluoroscopic motion data into three-dimensional (3D) in vivo biomechanical motion data.

Objective The aim of this study is to determine the in vivo biomechanical differences in patients with and without acute low back pain. Current dynamic imaging of the lumbar spine consists of flexion-extension static radiographs, which lack sensitivity to out-of-plane motion and provide incomplete information on the overall spinal motion. Using a novel technique, in-plane and coupled out-of-plane rotational motions are quantified in the lumbar spine.

Methods A total of 30 participants-10 healthy asymptomatic subjects, 10 patients with low back pain without spondylosis radiologically, and 10 patients with low back pain with radiological spondylosis-underwent dynamic fluoroscopy with a 3D-to-2D image registration technique to create a 3D, patient-specific bone model to analyze in vivo kinematics using the maximal absolute rotational magnitude and the path of rotation.

Results Average overall in-plane rotations (L1-L5) in patients with low back pain were less than those asymptomatic, with the dominant loss of motion during extension. Those with low back pain also had significantly greater out-of-plane rotations, with 5.5 degrees (without spondylosis) and 7.1 degrees (with spondylosis) more out-of-plane

Keywords

- low back pain

- lumbar strain

- spondylosis

- biomechanics

- kinematics

rotational motion per level compared with asymptomatic subjects.

Conclusions Subjects with low back pain exhibited greater out-of-plane intersegmental motion in their lumbar spine than healthy asymptomatic subjects. Conventional flexion-extension radiographs are inadequate for evaluating motion patterns of lumbar strain, and assessment of 3D in vivo spinal motion may elucidate the association of abnormal vertebral motions and clinically significant low back pain.
\end{abstract}

\section{Study Rationale}

Lumbar flexion-extension radiographs are commonly used to evaluate for abnormal motion and "dynamic" instability, but are of limited value due to the nature of two-dimensional (2D) static images at the end-ranges of sagittal plane motion. ${ }^{1-5}$

received

September 16, 2012

accepted

February 18, 2013

\section{Objective}

We used an in vivo fluoroscopic three-dimensional (3D) lumbar model to assess spinal motion in asymptomatic and symptomatic patients to determine the altered kinematics of lumbar strain associated with low back (c) 2013 Georg Thieme Verlag KG Stuttgart · New York
DOI http://dx.doi.org/ 10.1055/s-0033-1341640. ISSN 1663-7976. 




Fig. 1 Patient selection diagram. Convenience sample of 30 subjects was recruited with 10 subjects in each group based on the inclusion criteria. LBP, low back pain; MARM, maximal absolute rotational magnitude; POR, path of rotation.

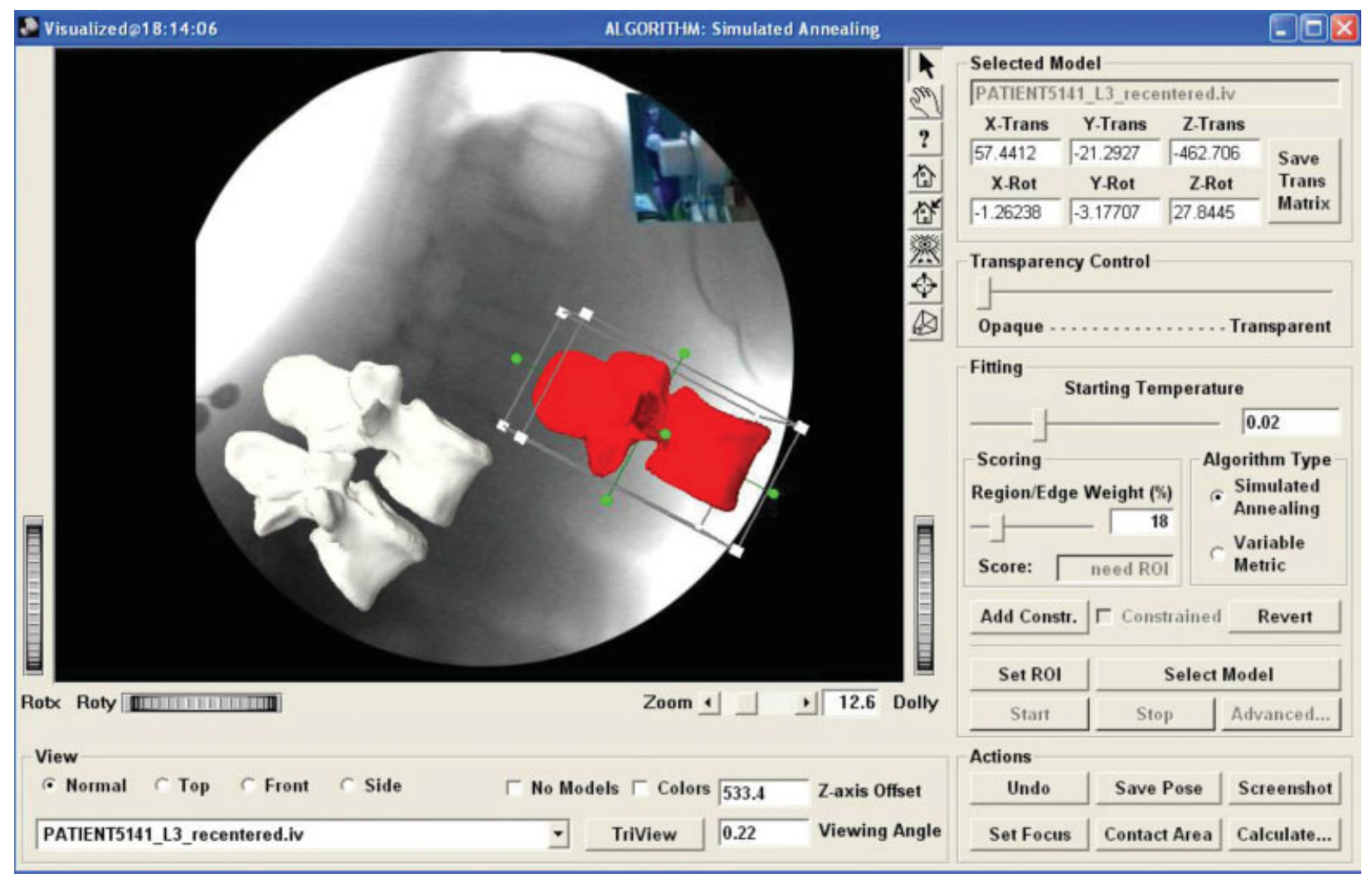

Fig. 2 Data window illustrating the 3D-to-2D image registration process. The vertebra contained in the box is placed over the appropriate vertebral silhouette and the "best fit" is achieved by initializing the global optimization simulated annealing algorithm. 


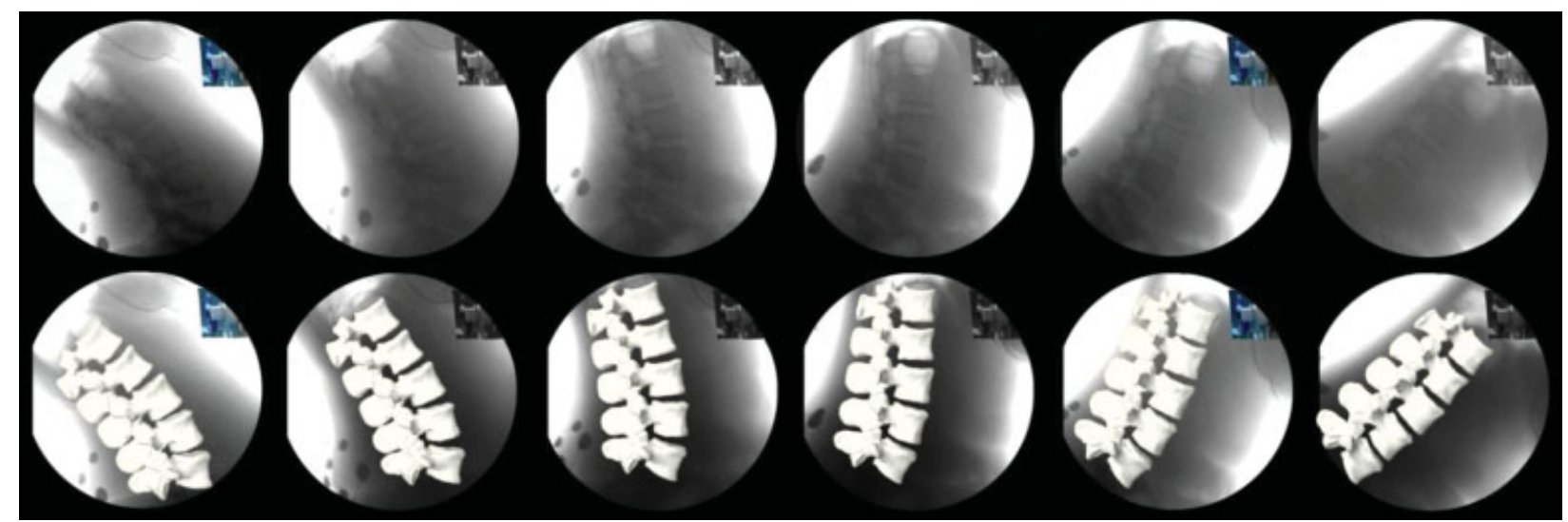

Fig. 3 Sample subject under fluoroscopic surveillance with image frames captured at full extension, 33\% of ROM, 66\% of ROM, and full forward flexion (top) with completed 3D-to-2D registration of image sequence (bottom).

pain in patients with and without radiological evidence of spondylosis.

\section{Methods}

Study Design: We present translational biomechanical study of a patient-specific 3D spinal motion model to determine differences in patients with and without acute low back pain.
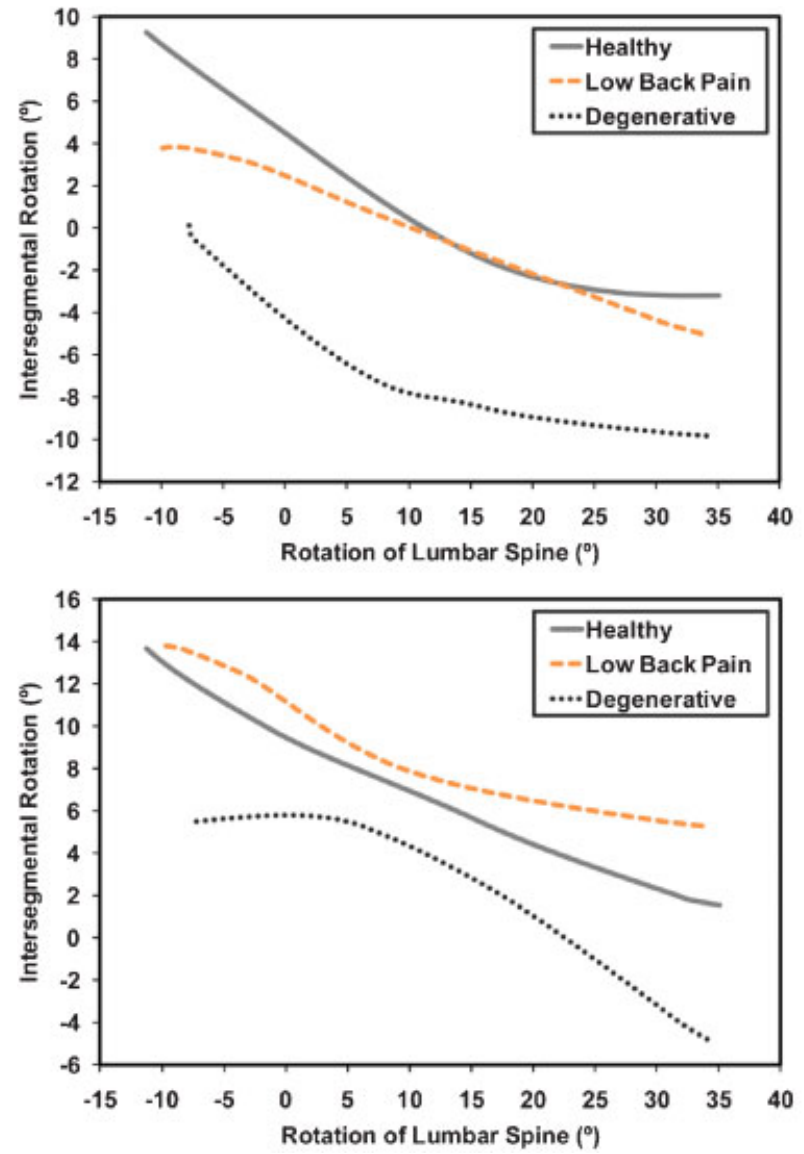

\section{Inclusion Criteria:}

- Group 1 was of asymptomatic subjects, never treated for low back pain.

- Group 2 was of acute low back pain, with a normal spine on radiology studies.

- Group 3 was of acute low back pain, with radiological findings of lumbar degeneration and spondylosis.

- Modic changes

- Degenerative disc disease with Schmorl nodes or disc bulging
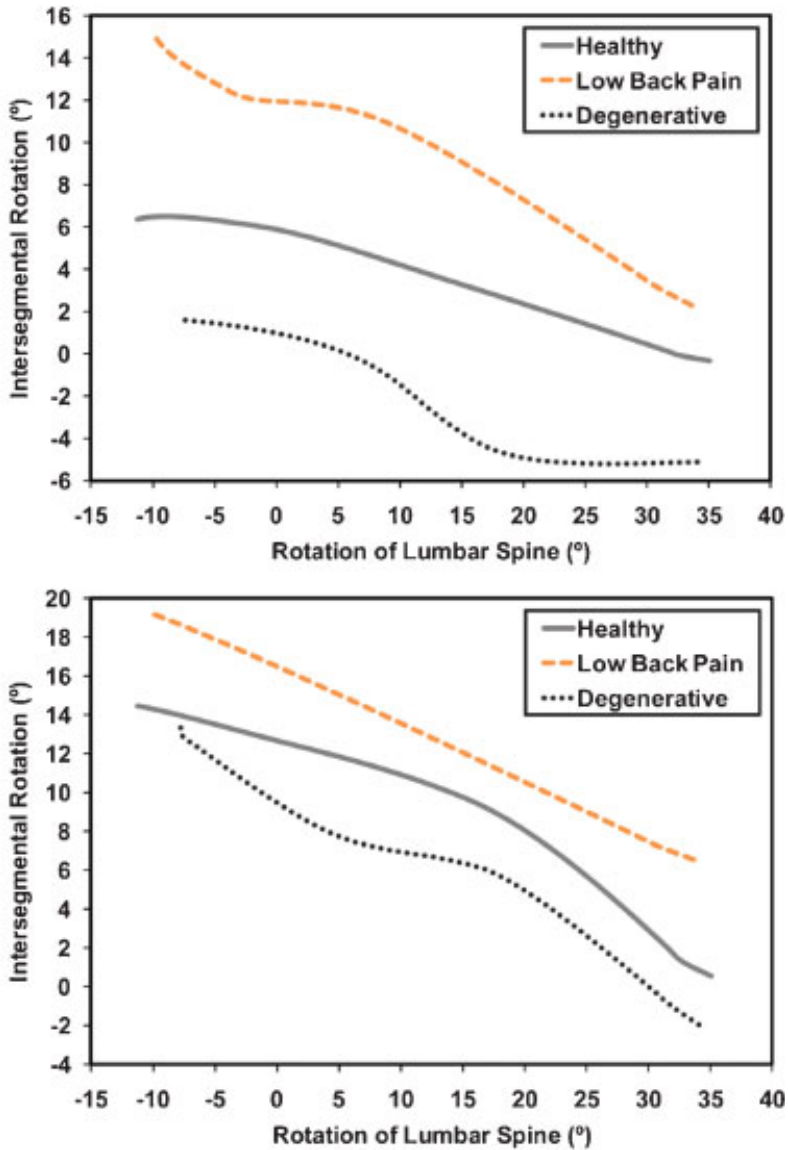

Fig. 4 Comparison of the intersegmental in-plane rotations relative to the average flexion plus extension of the lumbar spine at four levels (A, L1-L2; B, L2-L3; C, L3-L4; D, L4-L5). 
- Spinal canal or foraminal stenosis

- Disc osteophyte complexes

○ Facet spondyloarthropathy

\section{Exclusion Criteria:}

- Nondegenerative diseases

- Chronic low back pain

- Sensory loss

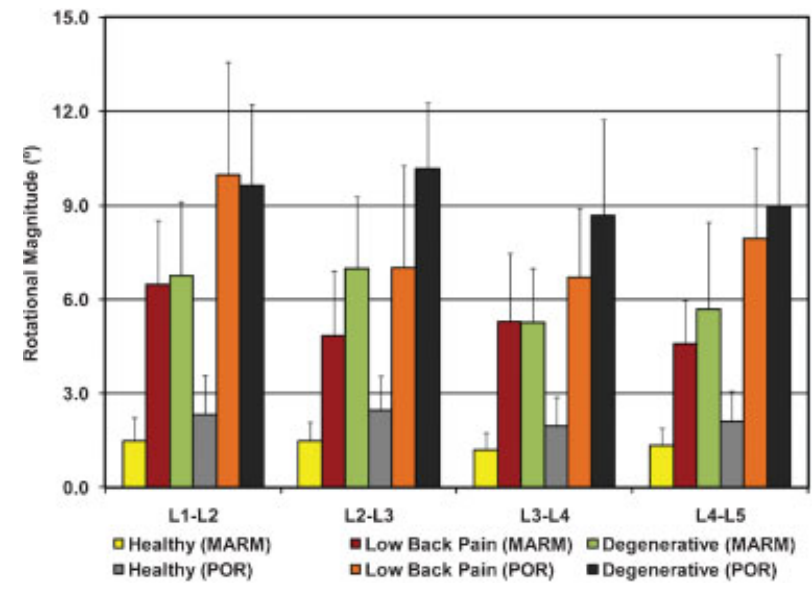

Fig. 5 The combined coupled axial rotation and lateral bending motions representing the average overall intersegmental out-of-plane rotations in three patient spine types derived using both the maximal absolute rotational magnitude (MARM) and path of rotation (POR) techniques.
- Motor weakness

- Prior lumbar spine surgery

\section{Patient Selection:}

- This study recruited a convenience sample of 30 subjects, 10 subjects in each group with an equal distribution of males and females except for Group 3

(-Fig. 1, -Table 1).

○ Healthy, asymptomatic subjects never treated for low back pain.

- Acute low back pain without radiological evidence of degeneration.

- Acute low back pain with radiological findings of lumbar degeneration and spondylosis.

\section{Translational Biomechanical Model:}

- Patient-specific spiral computed tomography scan was used to create 3D models of the L1 through L5 vertebrae.

- Model was overlaid with a local coordinate system assigned based on the Standardization and Terminology Committee of the International Society of Biomechanics 6 (-Fig. 2).

- Fluoroscopic video of flexion plus extension motion captured using a pulsed $\mathrm{x}$-ray output at 30 frames per second.

- A 3D-to-2D intensity-based image registration method was used to fit the 3D model to the fluoroscopic motion video capture ${ }^{7,8}$ (-Fig. 3 )

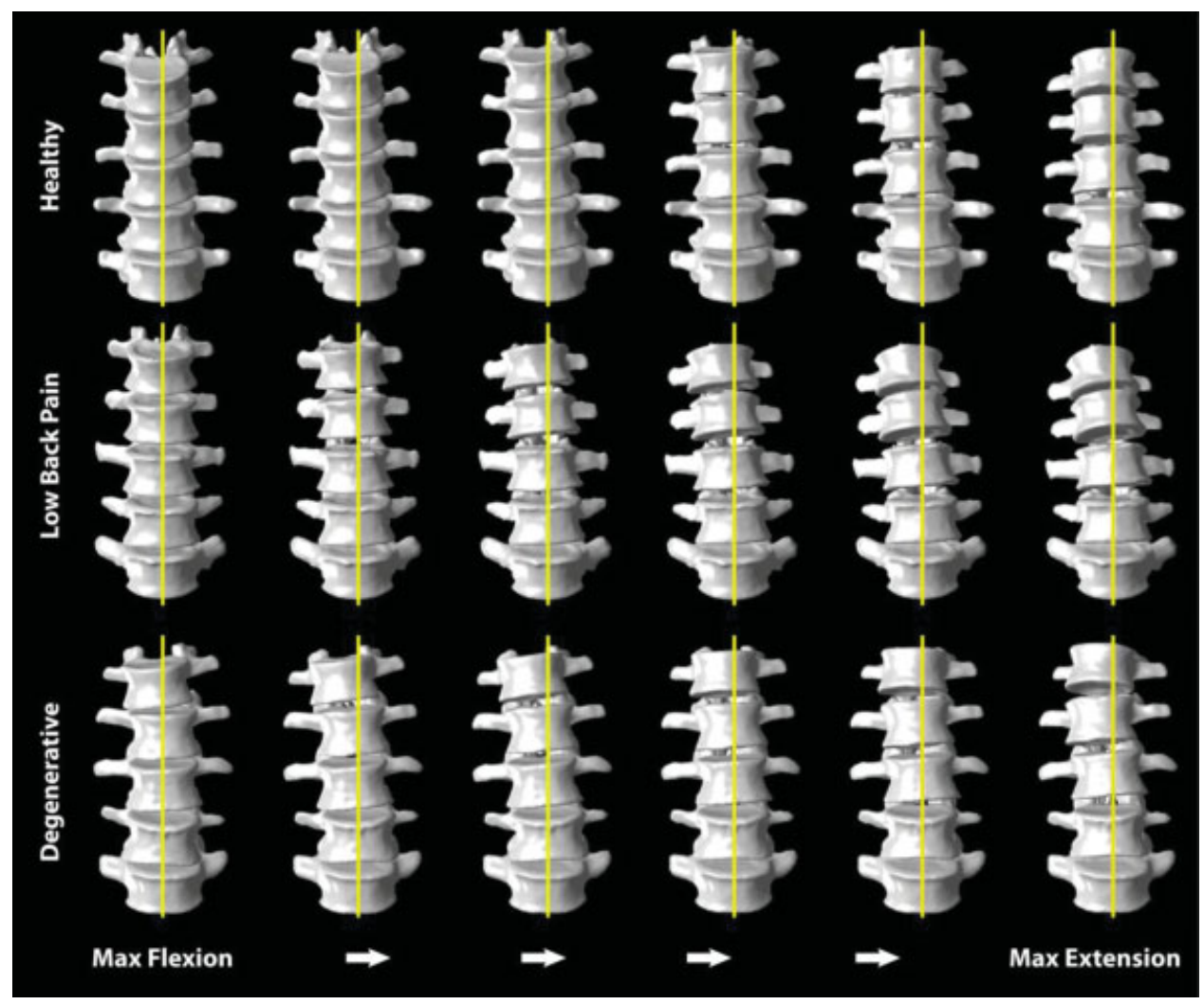

Fig. 6 A healthy spine, a spine with low back pain, and a degenerative lumbar spine moving from maximum flexion to maximum extension. The line bisecting the stationary L5 vertebrae helps visualize the increased out-of-plane movements in both low back pain and degenerative patients. 
10 Altered Spinal Motion in Low Back Pain Cheng et al.

Table 1 Patient characteristics

\begin{tabular}{|c|c|}
\hline & $N=30$ \\
\hline Age, years median (range) & $41.7(23-65)$ \\
\hline \multicolumn{2}{|c|}{ Group 1: Asymptomatic subjects, never treated for low back pain } \\
\hline Male, $n(\%)$ & $5(50)$ \\
\hline Spondylosis, $n(\%)$ & $0(0)$ \\
\hline \multicolumn{2}{|c|}{ Group 2: Acute low back pain with a normal spinal radiology studies } \\
\hline Male, $n(\%)$ & $5(50)$ \\
\hline Spondylosis, $n(\%)$ & $0(0)$ \\
\hline \multicolumn{2}{|c|}{ Group 3: Acute low back pain, with lumbar degeneration and spondylosis } \\
\hline Male, $n(\%)$ & $6(60)$ \\
\hline Spondylosis, $n(\%)$ & $10(100)$ \\
\hline
\end{tabular}

Table 2 Average in-plane range of motion for the lumbar spine from maximum flexion to maximum extension

\begin{tabular}{|c|c|c|}
\hline \multicolumn{3}{|c|}{ Flexion plus extension (degrees) } \\
\hline Type of spine & Mean \pm SD & Range of values \\
\hline Healthy & $46.6 \pm 10.8$ & $31.0-67.0$ \\
\hline Low back pain & $44.8 \pm 13.6$ & $20.0-62.0$ \\
\hline Degenerative & $42.5 \pm 10.3$ & $28.0-57.0$ \\
\hline
\end{tabular}

Abbreviation: SD, standard deviation.

Table 3 Average primary intersegmental in-plane rotation for all groups in the present study compared with data from previous literature

\begin{tabular}{|c|c|c|c|c|c|c|}
\hline \multirow[t]{3}{*}{ Spine level } & \multicolumn{6}{|c|}{ Flexion plus extension (degrees) } \\
\hline & \multicolumn{3}{|c|}{ Type of spine } & \multicolumn{3}{|c|}{ Previous literature (author, yr) } \\
\hline & Healthy & Low back pain & Degenerative & $\begin{array}{l}\text { Pearcy et al } \\
(1984)^{9}\end{array}$ & $\begin{array}{l}\text { Dvorák } \\
(1991)^{3}\end{array}$ & $\begin{array}{l}\text { White and } \\
\text { Panjabi }(1990)^{10}\end{array}$ \\
\hline L1-L2 & $11.8 \pm 3.2$ & $11.0 \pm 2.1$ & $10.8 \pm 2.6$ & $13.0 \pm 5.0$ & 11.9 & 12.0 \\
\hline L2-L3 & $9.6 \pm 3.0$ & $9.7 \pm 4.1$ & $9.9 \pm 4.2$ & $14.0 \pm 2.0$ & 14.5 & 14.0 \\
\hline L3-L4 & $12.2 \pm 4.5$ & $9.9 \pm 4.6$ & $11.1 \pm 3.9$ & $13.0 \pm 2.0$ & 15.3 & 15.0 \\
\hline L4-L5 & $13.1 \pm 3.8$ & $14.4 \pm 5.7$ & $10.7 \pm 3.6$ & $16.0 \pm 4.0$ & 18.2 & 17.0 \\
\hline
\end{tabular}

Note: Pearcy, Dvorák, and White/Panjabi motion values derived from normal asymptomatic volunteers.

Table 4 Average out-of-plane rotations from L1 to L5 derived using the MARM and POR methods

\begin{tabular}{|l|l|l|l|l|l|l|}
\hline \multirow{2}{*}{ Type of spine } & \multicolumn{5}{l|}{ Coupled out-of-plane rotations (degrees) } \\
\cline { 2 - 7 } & MARM & LB & Summation (AR + LB) & AR & LB & Summation (AR + LB) \\
\cline { 2 - 7 } & AR & $2.9 \pm 0.9$ & $5.5 \pm 1.9$ & $3.9 \pm 1.9$ & $4.9 \pm 1.4$ & $8.8 \pm 2.9$ \\
\hline Healthy & $2.5 \pm 1.1$ & 2.9 & $15.6 \pm 4.0$ & $16.1 \pm 5.3$ & $31.6 \pm 8.2$ \\
\hline Low back pain & $10.6 \pm 2.3$ & $10.6 \pm 3.2$ & $21.2 \pm 4.8$ & $19.2 \pm 7.0$ & $18.3 \pm 3.7$ & $37.5 \pm 8.6$ \\
\hline Degenerative & $12.2 \pm 5.1$ & $12.6 \pm 2.4$ & $24.7 \pm 6.7$ & & \\
\hline
\end{tabular}

Abbreviations: AR, axial rotation; LB, lateral bending; MARM, maximum absolute rotational magnitude; POR, path of rotation. 
- 3D rotational magnitudes were analyzed using maximum absolute rotational magnitude, calculated by finding the difference between any two increments representing the minimum and maximum motion observed, and the path of rotation of each functional spinal, tracked as it articulated upon the more caudal vertebrae.

\section{Results}

Patients with clinical and radiological findings achieved less overall in-plane rotation between L1 and L5 (-Table 2). Intersegmental in-plane rotations were not statistically different among groups, but motion about the flexionextension axis at L3-L4 and L4-L5 levels were altered in those with acute low back pain (-Table 3 ). In comparison with the asymptomatic group, those with acute low back pain had decreased values during extension with an overall loss of in-plane range of motion moving from full flexion to extension. Intersegmental lumbar spine rotation had similar rotational magnitudes but different patterns of rotation among the three groups (-Fig. 4). Analyses of the out-of-plane rotational motions indicate significantly more motion in those with low back pain compared with asymptomatic subjects (-Table 4, - Fig. 5). These out-of-plane movements were also indicated when viewing the flexion plus extension activity in the coronal plane (-Fig. 6).

\section{Discussion}

- Surgically relevant low back pain has a biomechanical etiology, originating when a loading stress is applied and exacerbated by abnormal motion or balance.

- Current radiological studies do not assess abnormal loading stress or motion.

- "Dynamic" flexion-extension radiographs are only static images obtained at end-ranges of a 2D sagittal plane.

- An in vivo fluoroscopic 3D lumbar model can be used to evaluate abnormal motion patterns in patients.

- Our study showed the differences in lumbar spinal motion in healthy asymptomatic subjects, patients with acute low back pain but without spondylosis, and patients with acute low back pain with radiological findings of spondylosis.

- Further work is needed to determine the utility of this advanced imaging technique to elucidate patterns of acute and chronic low back pain.

\section{Conclusions}

Using a technique involving video fluoroscopy and 3D-to-2D image registration, we were able to measure out-of-plane motions in the lumbar spine that are not detected with conventional lumbar flexion-extension radiographs. This will facilitate the understanding, assessment, and interpretation of clinically significant abnormal vertebral motions.

\section{Acknowledgments}

The research for this publication was supported by grant number 5R01AR055882 from the National Institute of Arthritis and Musculoskeletal and Skin Diseases (NIAMS) at the National Institutes of Health.

\section{Conflict of Interest}

All the authors have disclosed that they have no relevant financial relationships with the manufacturer(s) of any commercial product(s) and/or provider of commercial services discussed in this article.

Institutional Review Board approval as well as informed consent was obtained for all patients participating in this study.

\section{References}

1 Cholewicki J, McGill SM, Wells RP, Vernon H. Method for measuring vertebral kinematics from videofluoroscopy. Clin Biomech (Bristol, Avon) 1991;6(2):73-78

2 Dickey JP, Pierrynowski MR, Bednar DA, Yang SX. Relationship between pain and vertebral motion in chronic low-back pain subjects. Clin Biomech (Bristol, Avon) 2002;17(5): 345-352

3 Dvorák J, Panjabi MM, Chang DG, Theiler R, Grob D. Functional radiographic diagnosis of the lumbar spine. Flexion-extension and lateral bending. Spine (Phila Pa 1976) 1991;16(5): 562-571

4 Dvorák J, Panjabi MM, Novotny JE, Chang DG, Grob D. Clinical validation of functional flexion-extension roentgenograms of the lumbar spine. Spine (Phila Pa 1976) 1991;16(8):943-950

5 Harada M, Abumi K, Ito M, Kaneda K. Cineradiographic motion analysis of normal lumbar spine during forward and backward flexion. Spine (Phila Pa 1976) 2000;25(15):1932-1937

6 Li G, Wang S, Passias P, Xia Q Li G, Wood K. Segmental in vivo vertebral motion during functional human lumbar spine activities. Eur Spine J 2009;18(7):1013-1021

7 Mahfouz MR, Hoff WA, Komistek RD, Dennis DA. A robust method for registration of three-dimensional knee implant models to twodimensional fluoroscopy images. IEEE Trans Med Imaging 2003; 22(12):1561-1574

8 Liu F. Theoretical Modeling and Experimental Validation of In Vivo Mechanics for Subjects Having Variable Cervical Spine Conditions [dissertation]. University of Tennessee. Knoxville, TN: ProQuest/ UMI; 2007 (Publication No. AAT Thesis 2007b. L58)

9 Pearcy MJ, Tibrewal SB. Axial rotation and lateral bending in the normal lumbar spine measured by three-dimensional radiography. Spine 1984;9(6):582-587

10 White AA III, Panjabi MM. Clinical Biomechanics of the Spine. 2nd ed. Philadelphia, PA: Lippincott Williams \& Wilkins; 1990 
12 Altered Spinal Motion in Low Back Pain Cheng et al.

\section{Editorial Perspective}

The EBSJ reviewers congratulate Cheng et al on their radiographic study comparing lumbar intersegmental motion in three cohorts: a normal control group, a group with strain, and a group with radiographically manifest spondylosis-the latter two groups both with less than 3 months of symptoms. As pointed out in the latest AOSpine book, Measurements in Spine Care, ${ }^{1}$ the hope to find objectifiable and reproducible data to quantify back disorders through harmless, noninva- sive testing methods has frequently proven to be disappointing. This more sophisticated image analysis technique did show convincing differences in intervertebral motion patterns of the three groups. This technique questions previous attempts at measuring for abnormal motion patterns between lumbar vertebrae on lumbar standing flexion/ extension radiographs; perhaps they are simply too clumsy and inaccurate for meaningful analysis. 